\title{
Evaluation of time-limited trials among critically ill patients with advanced medical illnesses and reduction of nonbeneficial ICU treatments
}

Chang DW et al. JAMA Internal Medicine 2021; 181(6):786-794

M anche Patient*innen haben durch schwere Vorerkrankungen und fortgeschrittene funktionelle Einschränkungen eine von vornherein stark reduzierte Lebenserwartung. Kommt dann eine akute Erkrankung hinzu oder verschlechtert sich eine chronische Vorerkrankung, stellt sich uns oft die Frage nach der Sinnhaftigkeit intensivtherapeutischer Maßnahmen.

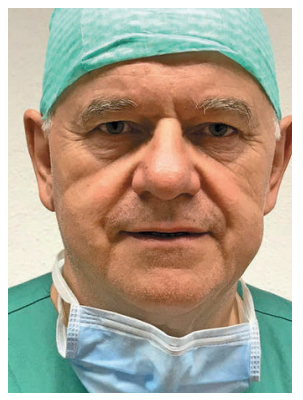
Von Prim. Univ.-Prof. Dr. Walter Hasibeder Leiter der Abteilung für Anästhesiologie und operative Intensivmedizin am Krankenhaus St. Vinzenz, Zams Dabei bewegen sich Intensivmediziner*innen und intensivmedizinisches Pflegepersonal häufig in einem Spannungsfeld zwischen medizinischer Indikation, Wertvorstellungen und dem mutmaßlichen Willen der Patient*innen sowie den Wünschen und Ängsten der Angehörigen.

In einer Studie aus den USA wurde bei 96 Patient*innen (mittleres Alter 63,6 Jahre), bei denen aufgrund der Schwere der Vorerkrankungen und vorbestehend stark eingeschränkten physiologischen Reserven eine intensivtherapeutische Behandlung als eher aussichtslos betrachtet wurde, ein sogenannter „limitierter Therapieversuch" auf der Intensivstation gestartet. Alle intensivmedizinisch durchgeführten Maßnahmen wurden in Bezug auf ihre Erfolgsaussichten ausführlich mit den Angehörigen diskutiert und ein medizinisch als sinnvoll erachteter "Therapiekorridor" im Sinne einer „Do-Not-Escalate(DNE)-Strategie" festgelegt. Im Verlauf der Intensivtherapie wurden mit den Angehörigen regelmäßig Therapierisiken, das klinische Ansprechen der Patient*innen auf gesetzte Maßnahmen sowie prognostische und medizinische Details zu gesetzten Maßnahmen besprochen. Die Ergebnisse wurden anschließend mit denen von 209 gematchten Patient*innen aus der Präinterventionszeit verglichen.
Im Vergleich zur Präinterventionszeit wurden nach Einführung eines als medizinisch sinnvoll erachteten Therapiekorridors weniger Patient*innen invasiv mechanisch beatmet (-12,9\%), die Anzahl der Nierenersatztherapien wurde reduziert $(-10,3 \%)$ und es wurden weniger häufig zentralvenöse Katheter angelegt ( $-32 \%)$. Die mediane Intensivaufenthaltsdauer wurde signifikant von 8,7 auf 7,4 Tage reduziert. Die Hospitalsmortalität war in beiden Gruppen mit 58,3 bzw. 58,4 Prozent annähernd gleich.

FAZIT für die Praxis: Auch in Österreich nimmt die Anzahl älterer und stark vorerkrankter Intensivpatient*innen deutlich zu. Oft erscheint dabei der Nutzen invasiver intensivtherapeutischer Maßnahmen fragwürdig. Für Angehörige ist es meist unmöglich, die prognostischen Aussichten und die Wertigkeit einzelner intensivmedizinischer Maßnahmen vollständig zu verstehen, und auch für Intensivmediziner*innen erschließt sich die Prognose der Patient*innen oft nicht sofort. Aus diesem Grund scheint das Festlegen eines medizinisch sinnvollen Therapiekorridors unter Einbeziehung der Angehörigen eine sinnvolle Maßnahme zu sein. Dafür müssen alle relevanten Informationen zu Vorerkrankungen und vorbestehenden funktionellen Einschränkungen eingeholt und die Schwere der aktuellen Erkrankung vollständig erfasst werden. Der festgelegte Therapiekorridor entspricht dabei einem zeitlich befristeten Therapieversuch. Innerhalb dieser Zeit wird sich die Prognose der Patientin/des Patienten aufgrund des klinischen Verlaufs eindeutig in eine positive oder negative Richtung entwickeln. Therapieziel-Änderungen hinsichtlich der Fortführung oder des Abbruchs intensivmedizinischer Maßnahmen können dann oft leichter getroffen werden.

\section{Effect of continuous infusion of hypertonic saline vs standard care on 6-month neurological outcomes in patients with traumatic brain injury - the COBI randomized trial}

Roquilly A, Moyer JD, Huet O et al. Jama 2021; 325:2056-2066

E twa 69 Millionen Menschen erleiden jährlich ein Schädel-Hirn-Trauma (SHT). Obwohl die Mortalität des SHT ständig sinkt, bleibt die Anzahl der Patient*innen mit Restschädigungen des Gehirns unverändert hoch (ca. acht Millionen/Jahr).

Eine adäquate Flüssigkeitstherapie ist ein wesentlicher Bestandteil der Versorgung von Patient*innen mit SHT. Die Vermeidung von Hypotonie und Hypovolämie verringert sekundäre Hirnschädigungen und verbessert die Prognose nach SHT. Dabei sollen hypertone Lösungen die Entwicklung eines traumatischen Hirnödems verhindern. In der vorliegenden Untersuchung wurden in einer multizentrischen, prospektiven randomisierten Studie die Effekte einer prophylaktischen Infusion 20\%iger $\mathrm{NaCl}$-Lösung $(n=185) \mathrm{im}$ Vergleich mit der üblichen Standardtherapie (Kontrollen; $n=185$ ) auf das neurologische Sechs-Monate-Outcome nach moderatem bis schweren SHT untersucht. Die $20 \%$ ige hypertone Kochsalzlösung wurde für mindestens 48 Stunden - bei erhöhtem Hirndruck auch länger - infundiert. Der primäre Outcome-Parameter der Studie war der "Glasgow Outcome Scale Extended"-Score (GOS-E Score; Bereich 1-8, wobei niedrigere Werte ein schlechteres funktionelles Ergebnis anzeigen). Weitere Outcome-Parameter waren die Entwicklung einer intrakraniellen Hypertension und die Halbjahresmortalität. 